\title{
Die Macht der Stimme
}

\section{Wie die Stimme wirkt, wie sie sich beeinflussen lässt - und was Stimmklang mit Wahrheit zu tun hat}

\author{
Prof. Carola Sonne \\ Trainerin für Stimme, Auftritt und Medien, Freiburg \\ www.venividivici-online.de
}

Erst kürzlich haben amerikanische Wissenschaftler in einer Studie nachgewiesen, dass Frauen an der Stimme eines Mannes erkennen können, wie stark der Mann ist. Was sie hörten, ohne ihn zu sehen, entsprach zuverlässig dem, was die Versuchsleiter über Messungen von Gewicht, Größe, Bizepsumfang und Händedruck festgestellt hatten. Interessant ist, dass die Forscher noch nicht wissen, welches Klangmerkmal denn nun die Stärke verrät. Die Tiefe scheint es jedenfalls nicht zu sein.

Gute Opernregisseure wissen um diesen Zusammenhang. Bewusst besetzen sie Rollen mit der passenden Stimme. Ein lyrischer Tenor klingt anders als ein Heldentenor. Der Tenor mit Schwert hat sozusagen klanglich mehr „Muskeln“ und klingt kämpferischer.

Welche Macht die Stimme hat, spüren wir an uns selbst: Wenige Worte können unser Gegenüber sympathisch oder abstoßend machen. Manche Stimmen verursachen Gänsehaut, andere ziehen uns in ihren Bann. Sicher ist: Jede Stimme ist so einzigartig und individuell wie ein Fingerabdruck. Was wir damit verbinden, ist im Gehirn gespeichert. Klangsignale werden wie Duft und Geschmack im Limbischen System emotional eingefärbt. Ob eine Stimme „stimmt“, entscheiden wir wie schon der Urmensch nicht durch Nachdenken, Vergleichen und Analysieren. Wir wissen es einfach - und zwar in Sekundenbruchteilen. Offensichtlich verrät die Stimme mehr, als wir uns vorstellen können - und bisweilen auch mehr, als uns recht ist.

Zunächst einmal ist der Stimmklang das Resultat der inneren wie äußeren Haltung. Die Klangerzeugung lässt sich also weitgehend physikalisch, physiologisch und psychologisch beschreiben und beeinflussen.

Erzeugt werden die Töne von den Stimmbändern. Die sind im Kehlkopf aufgehängt und klingen je nach Länge und Dicke höher oder tiefer. In der Regel klingt die Stimme einer großen Frau tiefer als die einer kleinen. Große Sopranistinnen sind so selten wie kleine Altistinnen. Die erzeugten Töne werden im Brustraum und in den Schädelhöhlen verstärkt und eingefärbt. Was die Stimmbänder schwingen lässt und die Resonanzräume öffnet, ist gut für die Stimme.

Schauen wir uns daraufhin einmal eine häufige Körperhaltung an: Die Schultern sind hochgezogen und fallen nach vorne, der Rücken ist nicht gestreckt und die Füße stehen nicht fest auf dem Boden - etwa durch hohe Absätze oder die Belastung nur eines Beins. Die Folge ist eine flache Atmung und der Verlust der tiefen Resonanzen. Zu hören ist überwiegend die leise Kopfstimme. Will man dennoch laut sprechen, wird die Stimme hoch und schrill.
Ein Blick auf Stimm-Profis zeigt, wie eine klangvolle, durchsetzungsstarke Stimme entstehen kann. Moderatorinnen, Dozentinnen oder Sängerinnen stehen fest und aufrecht. Ihr Blick ist zielgerichtet. Und noch etwas fällt auf. Menschen, die ihre Stimme gezielt einsetzen, beherrschen den Raum. Das heißt, sie stehen bewusst im Raum, fühlen dessen Größe und nehmen alle Menschen im Raum wahr. Etwa so, wie ein Dompteur in der Arena. Sie tun das von der ersten Sekunde ihres Auftritts bis zur letzten - egal, ob sie gerade eine Präsentation halten, einen Redebeitrag liefern oder nur anwesend sind.

So gesehen könnte es ganz einfach sein, mit einer besseren Haltung zu einer besseren Stimme und zu einer authentischen Wirkung auf andere zu gelangen.

Man beginnt einfach, auf dem ganzen Fuß zu stehen, die Brust aufzurichten, mehr Raum einzunehmen und den Blick auf ein Ziel zu richten. All dies wirkt unmittelbar auf den Stimmklang. Und es fühlt sich besser an, Menschen unverstellt mit dem gesamten körpersprachlichen Potential gegenüberzutreten. Tatsächlich aber genügt diese Belohnung selten, um das Verhalten nachhaltig zu ändern. Der Grund dafür liegt in der Effizienz des Gewohnten. Viele Elemente des individuellen Auftretens gehören zu einem aus der Kindheit mitgeschleppten Rollenverständnis. Früh entwickeln wir Strategien, uns durchzusetzen und Ziele zu erreichen. Am effektivsten tun wir das durch manipulative Kommunikation. Schon als Kinder setzen wir geschickt Haltungen und Stimmlagen ein, die bei den Eltern die gewünschte Reaktion auslösen.

Doch im Berufsleben ist die Strategie, die Papas kleinem Liebling die gewünschten Vorteile brachte, nicht mehr angemessen. Bestenfalls erreichen wir so ein Ziel. Aber wollen wir wirklich als süßes Mäuschen, als kleine Prinzessin oder aufsässiges Gör wahrgenommen werden?

Körperhaltung, Blick und Stimmklang senden bei jeder Begegnung ein nonverbales Beziehungsangebot aus. Und gerade weil es nonverbal ist, wirkt es viel stärker als die gesprochenen Worte. Und so reagieren die anderen auf die Haltung und nicht auf den Wortinhalt. Das kann im privaten Smalltalk durchaus akzeptabel sein. Im Berufsleben steht es dem Umgang auf Augenhöhe mit Kollegen, Mandanten oder Kunden entgegen.

Eine Frau, die mit piepsiger Stimme auftritt, appelliert entweder an den Beschützerinstinkt („Tu mir nichts, hilf mir!“) oder provoziert Aggression („Schlag mich! Ich wehre mich nicht!“). Eine hauchige Stimme dagegen kann ein „Paare Dich mit mir ...! “ vermitteln. Das umso mehr, wenn ein betont weibliches oder sexy Outfit zusätzliche Signale sendet. Ein schwerer Kommunikations-Unfall, wenn es der Sprecherin darum geht, als fachkompetent wahrgenommen zu werden. 
Viele Frauen stellen sich mit solchen ungewollten, aber aufmerksamkeitsstarken Botschaften ein Bein. Eine WeibchenBotschaft in Haltung und Stimme weckt eine ungewollte Erwartungshaltung. Welpenschutz oder Paarungsinteresse bei Männern, Ablehnung einer Konkurrentin bei Frauen.

Also heißt die entscheidende Frage: Wie vermeide ich ungewollte Botschaften? Oder: Wie vermeide ich es, von meiner Kompetenz abzulenken? Kurz gesagt müssen sich Gestalt und Stimme im Einklang befinden. Eine Stimme ist also nicht gut oder schlecht, sondern angemessen oder unpassend. Ob dieser Einklang besteht, spüren wir instinktiv. Sehen wir einen großen kräftigen Menschen, erwarten wir eine tiefe Stimme. Spricht er zu hoch, sind wir irritiert. Aber auch Alter, Gefühlsverfassung und Vitalität teilen sich in Nuancen des Stimmklangs mit. Weicht das Gehörte vom Gespeicherten und Erwarteten ab, beginnt es in uns zu arbeiten: Wieso klingt das jetzt anders als es klingen müsste? Hat der Wolf Kreide gefressen? Stimmt etwas nicht?

Eine gedachte Null-Linie, auf die sich empfundene Abweichungen beziehen, ist die sogenannte Indifferenzlage. Sie entspricht der „Kaustimme“, also der Stimme, in der wir wohlig „Mhh, das schmeckt gut“ sagen. Ein entspannter souveräner Mensch spricht in dieser Lage. Radio- und Fernsehmoderator(inn)en beeinflussen damit ihr Publikum positiv. Aus dieser „Standby“-Lage drücken Erregung und Anspannung die Stimme nach oben. Je weiter und je länger andauernd sich ein Mensch beim Sprechen von seiner Indifferenzlage entfernt, desto schwerer stößt er auf Vertrauen.

Durchsetzungsstark ist eine weibliche Stimme, wenn ihr in einem aufgerichteten Körper das Brustregister zur Verfügung steht. Unterstützt wird sie von einem fokussierten Blick. In der Unterhaltung gilt er dem Gegenüber. Bei größerem Publikum saugt er sich an einem imaginären Punkt in der letzten Reihe fest. Die Formel Körperklang plus Augen ermöglicht es, ohne extreme Anstrengung die der Situation angemessene Lautstärke und Durchsetzungskraft zu erreichen. Wer etwas energisch vertritt, begibt sich übergangslos aus der Indifferenzlage durch eine offene Haltung, fokussierten Blick und deutliche Diktion in den „Brustton der Überzeugung“. Man muss nicht andauernd so sprechen. Aber man muss der Stimme anhören, dass sie jederzeit auch „kämpfen“ könnte. So wie ein Rennwagen schon im Leerlauf hören lässt, dass er mehr Kraft hat als ein Kleinwagen.

Die innere Haltung wirkt über einen Effekt, der erst seit wenigen Jahren in seinen physiologischen Grundlagen verstanden wurde. Menschen besitzen, wie viele Tiere, spezialisierte Nervenzellen, die Haltung und Bewegung anderer spiegeln. Das heißt, sie zeigen ein Erregungsmuster, als würde selbst die Haltung eingenommen oder die Bewegung gemacht. Diese Spiegelneuronen erlauben uns, über den Weg des körperlichen Ausdrucks die Gefühle anderer wahrzunehmen indem wir sie fühlen.

Was jemand denkt, bestimmt sein Gefühl. Was er fühlt, führt zu Körperhaltungen und Spannungen. Die wiederum kopiert sein Gegenüber mit Hilfe seiner Spiegelneuronen. Es ist praktisch fast unmöglich, sich beim Anblick eines verkrampften Menschen zu entspannen. Und in Gegenwart einer niedergeschlagenen Person die gute Laune zu behalten, kostet Anstrengung.

Umgekehrt können wir es Gesprächspartnern mit unserer nicht verspannten Haltung und authentischen Stimme leicht machen, sich zu entspannen und sich auf unsere gewollten Botschaften zu konzentrieren.

Nun geht es in der Justiz ja oft um Wahrheitsfindung. Wo objektive Indizien fehlen, bleibt die Einschätzung dessen, was gesagt wird. „Stimmt“ eine Behauptung. Oder ist sie „unstimmig"? Bei einer Lüge lassen sich Diskongruenzen von Aussage, Stimmklang und Körpersprache feststellen. Der Mensch meint in diesem Fall nicht das, was er sagt. Schon das echte Lächeln lässt sich so vom falschen unterscheiden. Während beim echten die Muskulatur um die Augen arbeitet und zur Produktion sehr hoher Frequenzen beiträgt, ist beim falschen fast nur der Mund beteiligt. Ergebnis: Ein flacher, heller Klang, der im Gegensatz zum emotional fundierten Lächeln beim Gegenüber keine Glückshormone freisetzt. Die Lächelfalle ist eine der Ursachen, die gerade Frauen nonverbal immer wieder ihre Entschiedenheit untergraben und ihre Position verhandelbar erscheinen lassen.

$\mathrm{Ob}$ sich eine Lüge auf die momentane Aussage bezieht oder ob da im Klang eine chronische Lebenslüge mitschwingt, eine angeübte Unaufrichtigkeit, wie ein Dauerlächeln mit verkrampftem Unterkiefer, lässt sich nur feststellen, wenn man die Person länger beobachtet. Denn auch hier zählt wieder die innere Haltung: Sobald ein Mensch aus tiefster Überzeugung selbst glaubt, was er sagt, nehmen wir keine Lüge wahr.

Wenn auch noch nicht hinreichend erforscht ist, wie sich Kraft und viele andere persönliche Eigenschaften stimmlich mitteilen, bleibt doch eine beruhigende Erkenntnis: Einige Verhaltensänderungen ermöglichen unserer Stimme, unser Potential über den Klang mitzuteilen.

Wir können punkten, wenn wir unsere äußere Haltung optimieren.

Überzeugungskraft aber entsteht erst, wenn wir uns unserer inneren Haltung bewusst geworden und durchdrungen sind von unserer Botschaft. 